\title{
TERRASAR-X PAYLOAD DATA PROCESSING: RESULTS FROM COMMISSIONING AND EARLY OPERATIONAL PHASE
}

\author{
H. Breit ${ }^{1}$, B. Schättler ${ }^{1}$, T. Fritz ${ }^{1}$, U. Balss ${ }^{3}$, H. Damerow ${ }^{2}$, E. Schwarz ${ }^{2}$ \\ ${ }^{1}$ German Aerospace Center (DLR), Remote Sensing Technology Institute, Weßling, Germany \\ T: +49-8153-28-1369, E-Mail: Helko.Breit@dlr.de \\ ${ }^{2}$ German Aerospace Center (DLR), German Remote Sensing Data Center, Neustrelitz, Germany \\ ${ }^{3}$ Technical University Munich, Remote Sensing Technology Institute, Munich, Germany
}

\begin{abstract}
TerraSAR-X, the first national German radar satellite, was launched in June 2007. It carries an X-band high-resolution synthetic aperture radar instrument featuring Stripmap, ScanSAR and, particularly, Spotlight imaging in a variety of different polarization modes. The mission completed its commissioning phase (CP) in December 2007, the provision of the SAR products for both the scientific and commercial user community was started in January 2008.

One central TerraSAR-X element on ground is the payload ground segment PGS. From the beginning of the mission, PGS was nominally operated. About ten thousand data takes were already acquired and processed in 2007 , not only for SAR verification and calibration purposes, but also for the operational ground segment validation.

This paper provides the commissioning and early operational phase results from the SAR payload data processing perspective addressing data reception and SAR processing. Specifically the tuning and adjustment of the TerraSAR-X Multi-Mode SAR Processor TMSP to meet the inorbit data characteristics and to optimize the SAR focusing results is addressed. Relevant issues are the high-bandwidth chirp replica processing, side lobe suppression, Doppler frequency determination, processor normalization and phase-preservation.
\end{abstract}

\section{Index Terms - TerraSAR-X, SAR, TMSP}

\section{INTRODUCTION}

The main objectives of the $\mathrm{CP}$ were the calibration and verification of the entire SAR system in order to achieve the specified SAR image and product quality as well as the operationalization and validation of the ground segment functionalities. Due to comprehensive system tests performed pre-launch including both the ground and space segment, the TerraSAR-X mission experienced a smooth transition into the CP. Thus, from the first days of the mission the focus of activities could be straightened to the processing of SAR payload data acquired in space.

\section{PAYLOAD GROUND SEGMENT OVERVIEW}

Within the TerraSAR-X ground segment the PGS performs SAR payload data reception, processing, archiving and dissemination. These tasks are organized as two workflows, the request and SAR workflow, respectively as depicted in Figure 1. The request workflow starts with a user order entered via EOWEB, processed by PGS ordering and production control and submitted to the mission planning entity of the mission operations segment MOS. Subsequently MOS commands the acquisition and the SAR payload data are downlinked to DLR's ground station in Neustrelitz. Here, the SAR data workflow starts with decryption and the SAR raw data are systematically processed to L0 archival products by the Processing System TMSP. In contrast to the data-driven L0-screening approach, the TMSP generates $\mathrm{L} 1 \mathrm{~b}$ products on user request. The $\mathrm{L} 1 \mathrm{~b}$ product is finally transferred to the archive for temporary storage and delivery to the user.

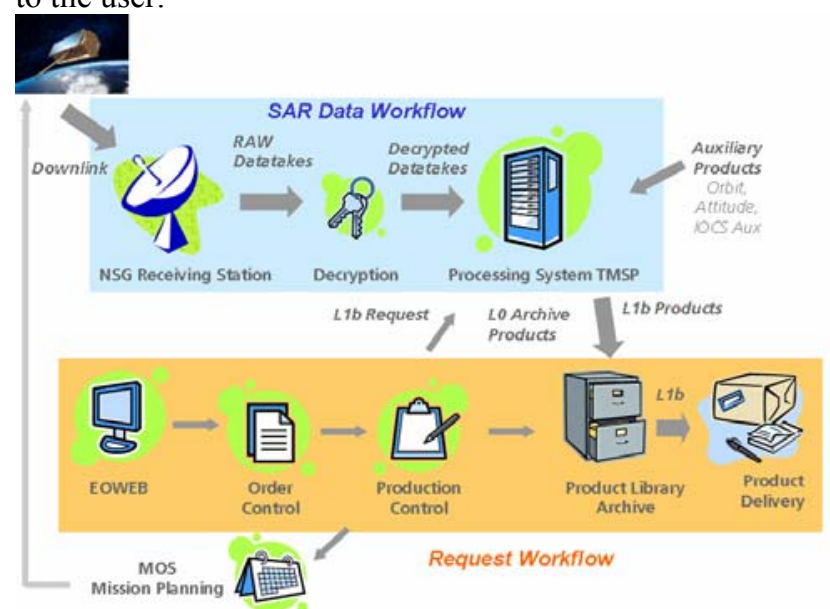

Figure 1: Schematic Payload Ground Segment workflow overview (courtesy: W. Balzer).

\section{TERRASAR MULTI-MODE SAR PROCESSOR}

The TMSP consistently generates phase-preserving [7], [8] single-look slant-range complex (SSC) data sets from the imaging modes Stripmap, ScanSAR and Spotlight for all specified polarization modes. This is achieved by a hybrid 
focusing kernel based on chirp scaling algorithm variants [2]. Derivation of multi-look detected products (MGD, GEC and EEC) [1] is based on SSCs as an interim production stage, depicted in Figure 2. The integration of a DEM data base supports the high-precision processing in terms of radiometry and geometry.

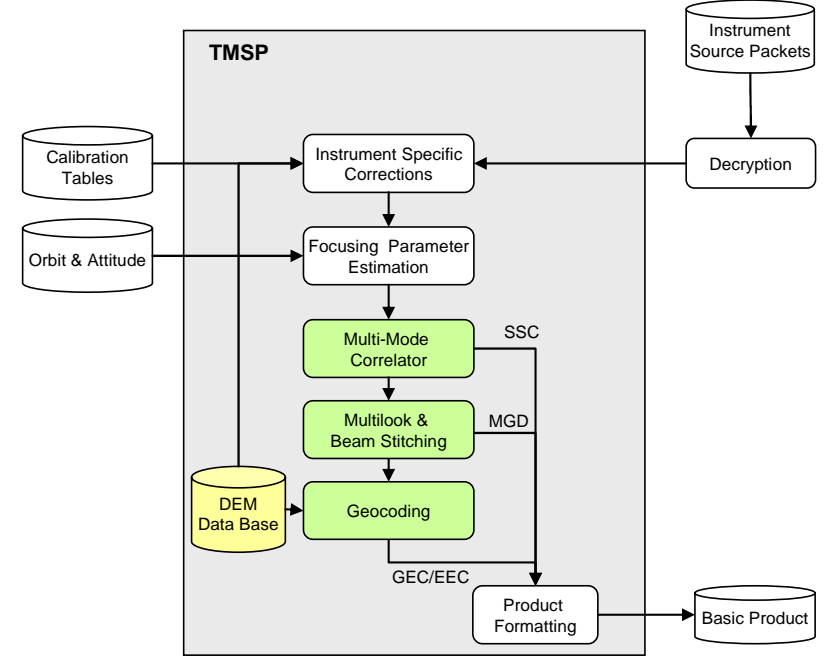

Figure 2: TMSP processing concept overview

\section{SAR PROCESSING ASPECTS}

Integrated digital elevation model:

Besides the application of 1" and 3" DEM data for precise orthorectification (EEC product [1]), the determination of azimuth FM rates, the projection of the elevation antenna pattern on the earth's surface and the derivation of the geometric Doppler centroid utilize a coarse 10" DEM.

Gain bean stripFar_o85 channel HH - SRA

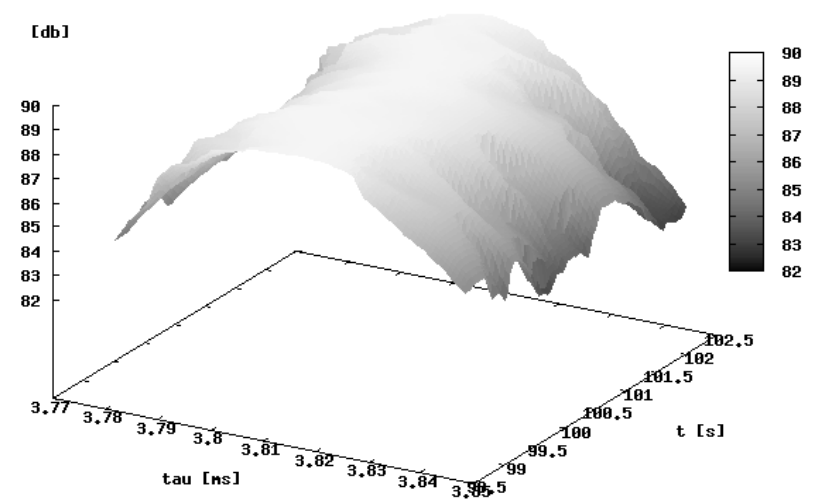

Figure 3: Example of an elevation gain antenna pattern illuminating a $30 \mathrm{~km} \times 30 \mathrm{~km}$ part of the earth's surface in an alpine area. The gain values are represented in slant range geometry $(t, \tau)$ and the gradients of the pattern are noticeable modulated by the underlying topography.
Gain Normalization:

Processor normalization ensures that the processing gain is independent of the range spread, chirp length, azimuth aperture length, PRF, range sampling frequency, imaging mode, processed bandwidth, applied weighting and output sampling. The normalization concept has been validated by corner reflector measurements and radiometrical seamless mosaics of rainforest images acquired with different SAR modes [5]. Due to the mode and acquisition parameter independent processor gain a single unique absolute calibration constant is obtained [4].

Doppler centroid estimation:

The Doppler centroid is estimated from the SAR signal data itself (baseband Doppler) an in addition derived from the attitude information (geometric Doppler). Fusion of both estimates leads to the Doppler centroid used during processing. Baseband and geometric Doppler values show a good alignment, Figure 4. The margins expected from the Total Zero Doppler Steering [6] are met and the suitability of the applied steering law is proven. The remaining absolute Doppler is found to be within $+/-120 \mathrm{~Hz}$. The signal based estimates verify very well the squint angles derived from the satellite's Attitude and Orbit Control System (AOCS) data. For TerraSAR-X a Doppler centroid of $80 \mathrm{~Hz}$ corresponds to a squint angle of about $0.01^{\circ}$.
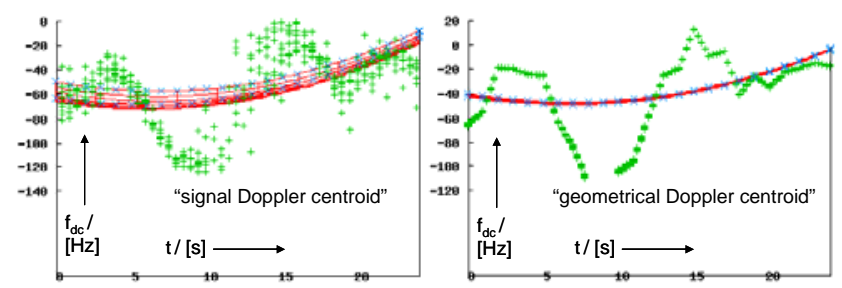

Figure 4: Comparison of baseband Doppler estimates (left) and the AOCS derived geometrical Doppler values (right) of a 24 s duration data take.

Range reference function determination:

A spectral analysis of a reconstructed chirp replica reveals that a simple polynomial fit is an inadequate model to describe the chirp signal being affected by non-linearities in the system transfer functions of the instrument's transmit and receive electronics, i.e. up / down conversion. The power level of the mirror-frequency chirp and other chirp components is about 20 to $25 \mathrm{~dB}$ below the nominal chirp.

In high contrast parts of a SAR image as depicted in Figure 5 strong scatters leave objectionable range artifacts of several kilometers length, i.e. the chirp length. Applying the reconstructed chirp replica itself the energy of all chirp components is focused to the scatters' true locations, see Figure 6. 


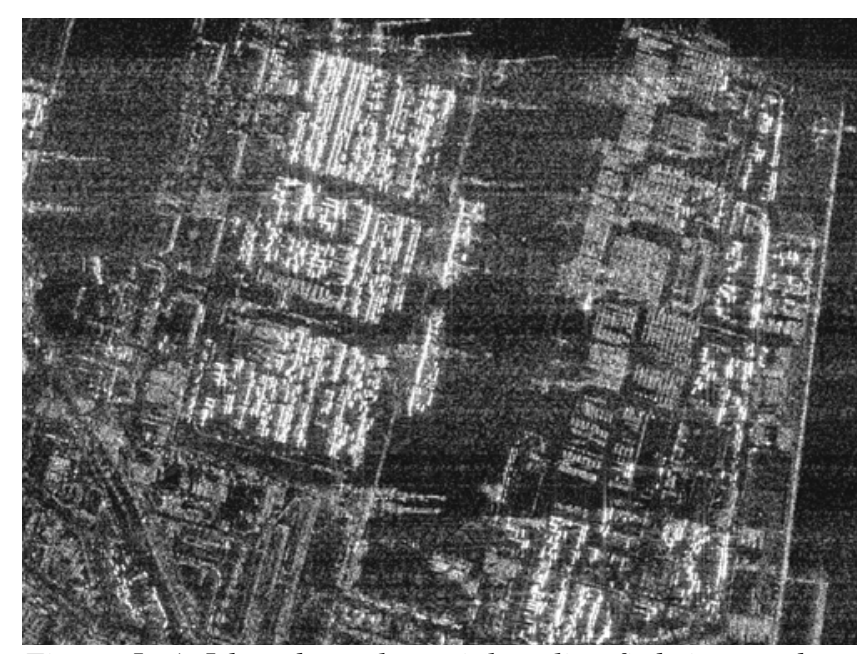

Figure 5: A 5th order polynomial replica fit being used as range reference function does not focus all range signal components. The given $2 \mathrm{~km}$ (az.) x $1.4 \mathrm{~km}$ (rg.) image detail depicts a container terminal located at Yokohama, Ja-

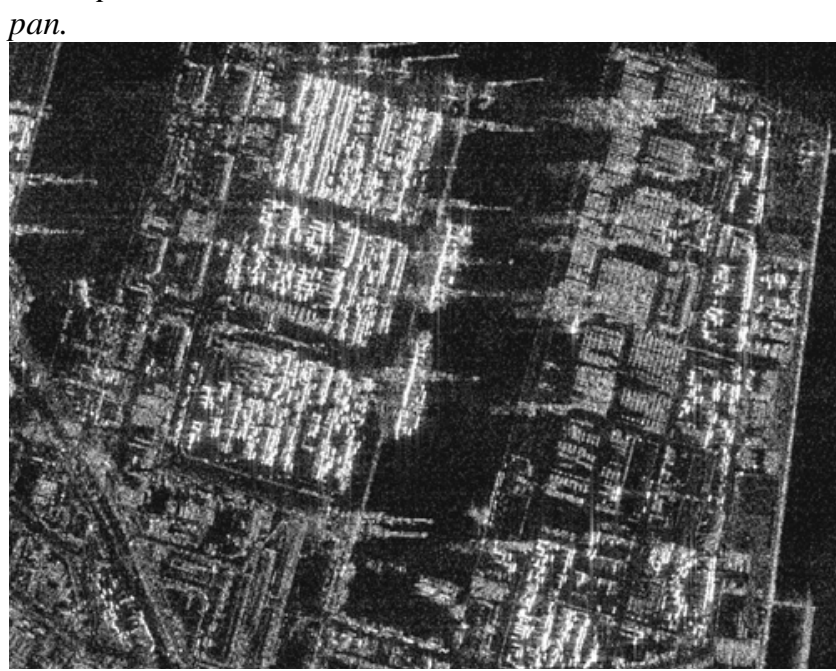

Figure 6: Direct use of a digital replica drastically improves the range focusing quality.

\section{Side lobe suppression:}

Weighting of the complex signal spectrum by a Hamming like window function,

$$
W(f)=\alpha+(1-\alpha) \cdot \cos (2 \pi f / B) \cdot \operatorname{rect}(f / B),
$$

suppresses side lobes on the expense of resolution. In contrast to the determination of an optimal range focusing reference function the trade-off between preferably high geometrical resolution on the one hand and an acceptable image degradation in terms of peak and integrated side lobe ratios (PLSR, ISLR), signal to azimuth ambiguity ratio (SAAR) and signal to noise ratio (SNR) one the other hand is always a compromise.

After inspection of the first high resolution TerraSAR$\mathrm{X}$ images processed with a pre-launch defined side lobe suppression of $\alpha=0.75$ (Figure 7) it seemed to be worthwhile reconsidering this parameter setting.

A weighting factor of $\alpha=0.60$ is a good compromise: Compared to the initial setting, PSLR improves by $10 \mathrm{~dB}$ and 1-D ISLR by $3.5 \mathrm{~dB}$. In contrast to range where the resolution is decreased by the theoretical value of $18 \%$ the effect is mitigated in azimuth by an increased processing bandwidth $(+11 \%)$ in Stripmap mode. A total compensation is achieved in HS Spotlight mode $(1.1 \mathrm{~m})$ by extended azimuth beam steering. In SL Spotlight mode the azimuth resolution is even improved $(1.7 \mathrm{~m})[1]$.

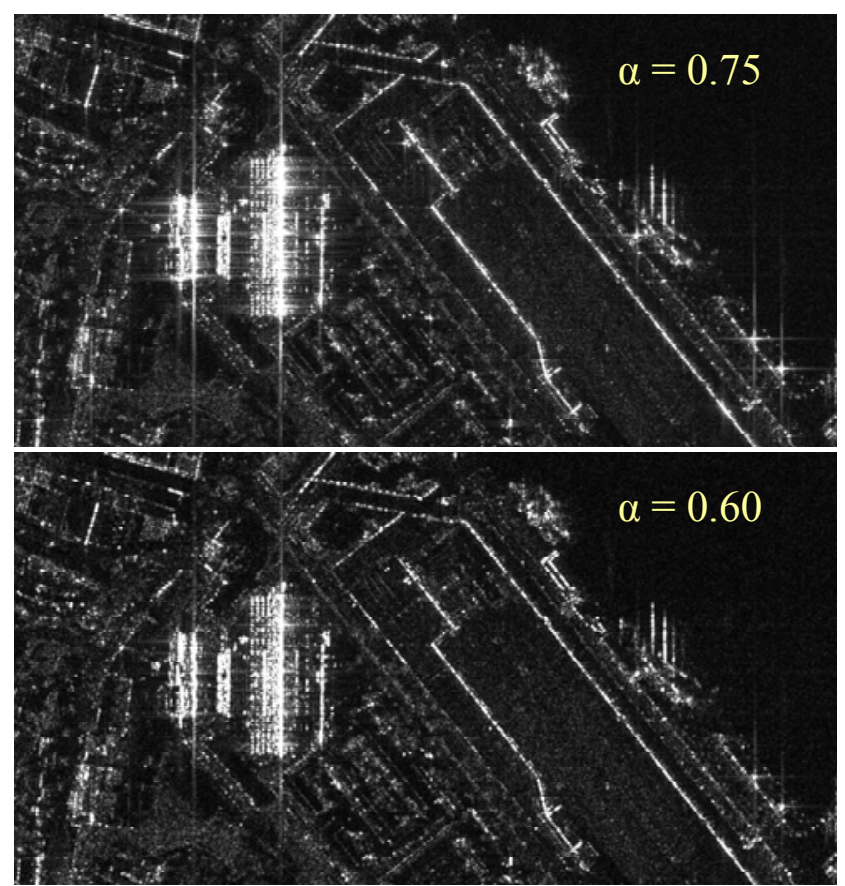

Figure 7: Detail of a $300 \mathrm{MHz} \mathrm{HS}$ image. The slightly stronger weighting effectively drops the unwanted side lobes especially in urban and industrial areas.

\section{Spotlight mode processing:}

TerraSAR-X provides two sliding Spotlight imaging configurations, the $S L$ mode with $1.7 \mathrm{~m}$ azimuth resolution, covering $10 \mathrm{~km}$ by $10 \mathrm{~km}$ and the HS (High Resolution Spotlight) mode with $1.1 \mathrm{~m}$ az.-resolution and an azimuth scene extend reduced to $5 \mathrm{~km}$. In addition to the standard $150 \mathrm{MHz} \mathrm{HS}$ mode there is an option for $300 \mathrm{MHz}$ on the expense of range swath width.

The $300 \mathrm{MHz}$ HS mode image in Figure 8 depicts the calibration test site Oberpfaffenhofen. The scene comprises signatures of transponders as well as of small reflectors hand-held by the participants of the "First TerraSAR-X Image" anniversary event, June 19, 2008.

The azimuth illumination conditions and therefore the spectral properties of this acquisition at an incidence angle of $56^{\circ}$ are illustrated in Figure 9. The dashed green line represents the Doppler centroid while the black parallelo- 
gram indicates the instantaneous processed bandwidth of $2800 \mathrm{~Hz}$. The slope corresponds to the commanded azimuth steering rate. The illuminated phase histories of the very first and very last target are shown in blue, respectively. The slopes correspond to the targets' FM rates. The zeroDoppler crossings mark the final azimuth extent (thick red part of the $x$-axis) of the focused image.

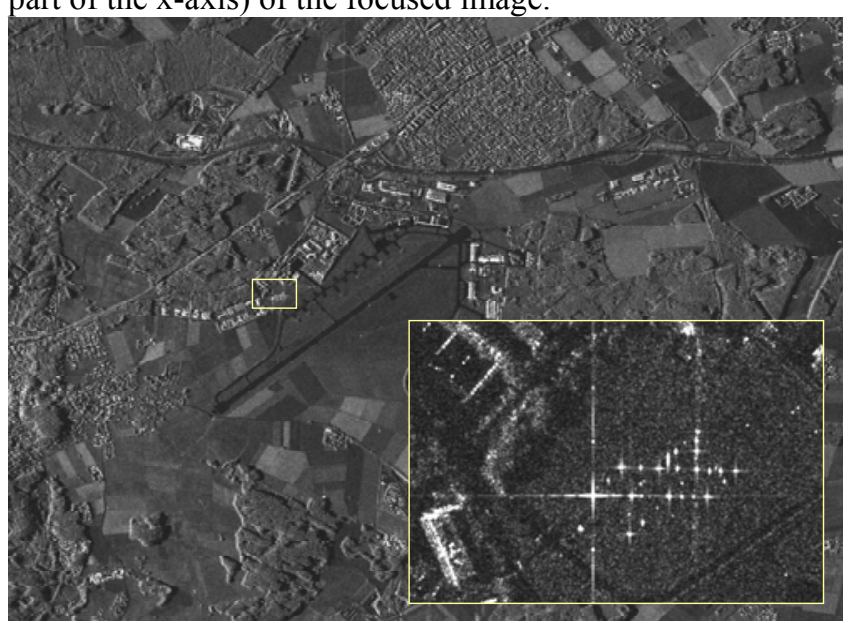

Figure 8: TerraSAR-X $300 \mathrm{MHz}$ High Resolution Spotlight image with an extent of $5.4 \mathrm{~km}(\mathrm{az}) \times 5.8 \mathrm{~km}(\mathrm{rg})$ covering the Oberpfaffenhofen calibration test site.

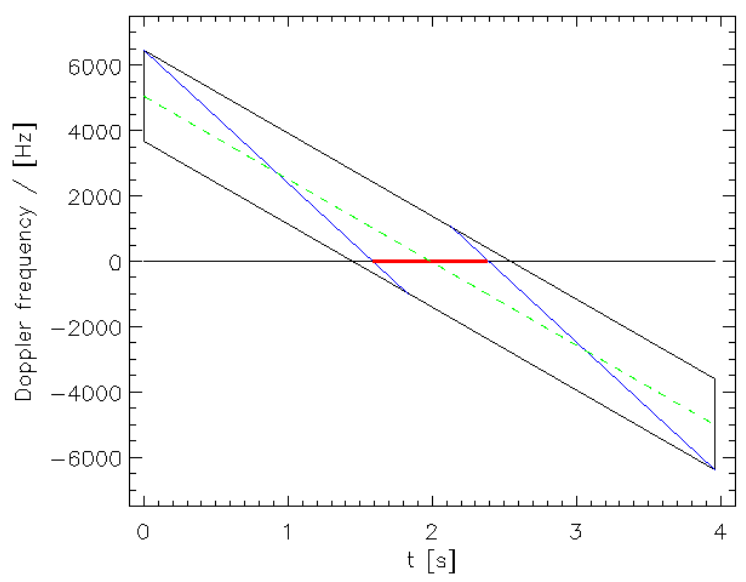

Figure 9: Spectral properties of the HS acquisition given as azimuth time versus instantaneous Doppler frequency plots.

Measurements on a transponder signature in the single look complex image result in an azimuth resolution of $1.08 \mathrm{~m}$ and a slant range resolution of $0.60 \mathrm{~m}$ and PSLR values ranging from $-29.7 \mathrm{~dB}$ to $-31.5 \mathrm{~dB}$. This is in accordance with the specification and confirms the $\mathrm{CP}$ results.

Polarization:

TerraSAR-X provides a dual-polarization capability with only one receiver channel using pulse by pulse polarization toggling. The TMSP corrects the time shift between polarization layers. The experimental quad polarization mode re- quires the switched-on redundant receiver as a second channel. Figure 10 gives an example of a color coded composite of the two image layers of a dual-pol. Stripmap product.

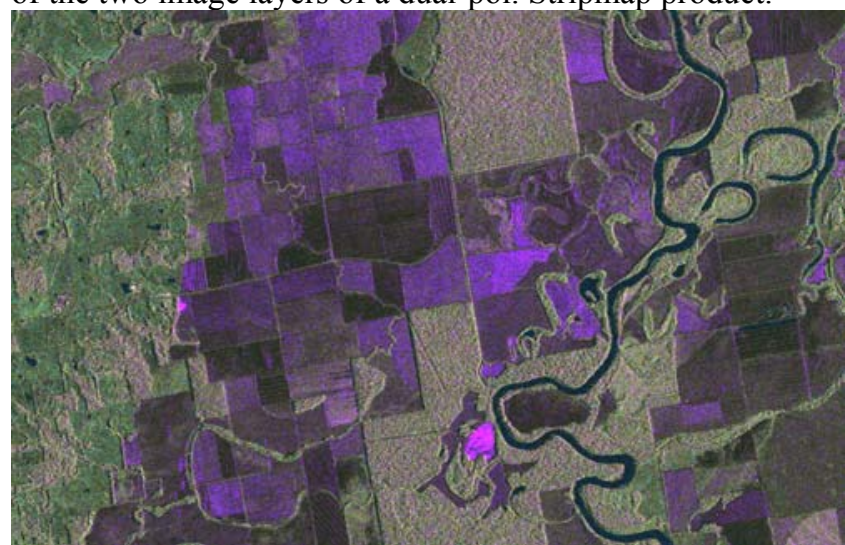

Figure 10: Detail (9 $\mathrm{km} \times 6 \mathrm{~km}$ ) of a dual-polarization Stripmap image. Color code: R: HH, G: HV, B: |HH-HV|.

\section{ACKNOWLEDGEMENT}

The authors would like to thank M. Stangl and M. Völker (EADS/Astrium) for fruitful discussions and valuable suggestions on the topic of range reference function determination.

\section{REFERENCES}

[1] TX-GS-DD-3302 TerraSAR-X Basic Product Specification

[2] M. Stangl, R. Werninghaus, B. Schweizer, C. Fischer, M. Brandfass, J. Mittermayer, H. Breit: "TerraSAR-X Technologies and First Results", IEE Radar, Sonar \& Navigation, 153 (2), pp. 86 $-95,2006$

[3] J. Mittermayer, R. Metzig, U. Steinbrecher, C. Gonzalez, D. Polimeni, J. Böer, Y. marwan, J. Márquez Martínez, S. Wollstadt, D. Schulze, A. Meta, N. Tous-Ramon, C. Ortega Miguez: "TerraSAR-X Instrument, SAR System Performance and Command Generation", Proc. of IGARSS 2008, Boston, USA.

[4] M. Schwerdt, B. Bräutigam, M. Bachmann, B. Döring, D. Schrank, J. Hueso Gonzalez: "Terrasar-X Calibration Results", Proc. of IGARSS 2008, Boston, USA.

[5] T. Fritz, H. Breit, B. Schättler, M. Lachaise, U. Balss, M. Eineder: "Terrasar-X Image Products: Characterization and Verification", Proc. of IGARSS 2008, Boston, USA.

[6] H. Fiedler, E. Boerner, J. Mittermayer, G. Krieger: "Total Zero Doppler Steering - A New Method for Minimizing the Doppler Centroid", IEEE Geosience and Remote Sensing Letters, Vol. 2, Iss. 2, pp. 141 - 145, April 2005.

[7] M. Eineder, N. Adam, R. Bamler, N. Yague-Martinez, H. Breit: "TerraSAR-X Spotlight SAR Interferometry". Submitted to IEEE, Transactions on Geoscience and Remote Sensing. 2008.

[8] N. Adam, M. Eineder, N. Yague-Martinez, R. Bamler: "High Resolution Interferometric Stacking with TerraSAR-X”, Proc. of IGARSS 2008, Boston, USA

[9] TerraSAR-X Image Products: Characterization and Verification. EUSAR 2008 Conference, Friedrichshafen, Germany, 2008. 